\title{
Varied treatment options enhance patients' survival of pulmonary embolism
}

The treatment of pulmonary embolism must be individualized to each patient's clinical condition. This condition includes the very risk factors that were, in part, responsible for the thrombotic event itself.

Systemic heparinization is the treatment of choice for most patients with pulmonary embolism. Sometimes, anticoagulation is contraindicated. When this situation occurs, the placement of an inferior vena caval filter becomes necessary. Likewise, if a severe hemorrhagic complication has occurred from anticoagulation therapy, then anticoagulants must be discontinued and placement of a filter in the vena cava is indicated.

Other situations require the consideration of interruption of the vena cava. These rare situations include the patient who has documented recurrent pulmonary embolism despite adequate anticoagulation; the patient who requires a surgical embolectomy because anticoagulation treatment is contraindicated postsurgically; the patient who has had a massive embolism and who is at risk of a recurrence; the patient with septic emboli from pelvic or lower extremity septic thrombophlebitis; and the patient with paradoxic emboli to the arterial circuit via a patent foramen ovale.

Most often, the transvenous method for interruption of the vena cava is used because it requires no general anesthetic; mortality and morbidity occur considerably less as well. The Greenfield filter is a common device used in this instance. However, the bird's nest filter is a new transvenous device that holds promise for the prevention of recurrent embolism without obstructing the inferior vena cava.

Another approach, thrombolytic therapy, is reserved for patients who have had a massive pulmonary embolic event with subsequent shock or severe hypoxemia. Open-chest pul- monary embolectomy while the patient is on cardiopulmonary bypass support and percutaneous transvenous embolectomy are alternative treatment measures for a rare, select group of patients. These procedures can be considered for a patient with acute, massive pulmonary embolism that is usually documented angiographically. Such a patient usually has persistent hemodynamic instability despite optimal medical therapy, or has an absolute contraindication to anticoagulation therapy.

It is the former type of patient about whom Drs Waters and Stanley report, beginning on page 125 in this issue of the JAOA. Despite having received streptokinase therapy, a 55year-old man had a massive pulmonary embolus that developed after thrombolytic therapy was begun. This patient was treated successfully with open pulmonary embolectomy supported with cardiopulmonary bypass. Whenever attempted, embolectomy should be accompanied by interruption of the inferior vena cava.

In the acute embolic setting, the mortality rate for open-chest cardiopulmonary bypasssupported embolectomy remains more than $50 \%$. Conversely, there is another rare group of patients who have chronic, recurrent thromboembolic pulmonary hypertension and cor pulmonale, where open-chest pulmonary embolectomy, or in actuality pulmonary endarterectomy, can produce remarkable systemic relief. This approach has a low incidence of surgical mortality. Hemodynamic improvement also occurs in carefully selected patients. The mortality associated with this procedure seems very low, particularly in institutions that specialize in treating such patients.

Pulmonary embolism remains a major cause of morbidity and mortality in the United 
States. A keen clinical suspicion for the presence of this disorder is necessary to reduce the morbid impact of this clinical manifestation of deep venous thrombosis. Preventing venous thrombosis remains the single most important way of reducing the frequency of pulmonary embolism. Newer methods of deep venous thrombosis prophylaxis, which deserve our attention, include the use of graded compression elastic stockings, intermittent pneumatic compression of the lower extremities, and a combination of these modalities, along with the subcutaneous use of heparin.

With these preventive measures and treatment options at our disposal, our patients' survival is considerably enhanced.

GILBERT E. D'ALONZO, DO

Professor of Medicine

Temple University Medical Center

Philadelphia, $\mathrm{Pa}$

\section{Type A personality may strike at the heart of CAD}

Since the 1970 s, the association of type A personality - characterized by hard-driving, competitive, aggressive, hurried behaviors - with myocardial ischemia has been repeatedly investigated, but often with conflicting results. Although this global type A personality has not been consistently correlated with myocardial or coronary artery disease (CAD), components of type A behavior, specifically anger and hostility, may prove to be the "silver bullet" in this personality-myocardial connection.

Investigators at the Uniformed Services University of Health Sciences in Bethesda, Md, reported on a similar connection at the summer meeting of the American Psychological Association held in San Francisco. Specifically, they studied three groups of patients with CAD. The CAD was assessed in each of the three groups by use of echocardiography, a thallium exercise test, or an ambulatory Holter monitor, respectively. Patients were classified as having a defensive-hostility, high-hostility, repressive-hostility, or a low-hostility coping style.

Those patients classified as defensively hostile had the highest mean scores for ventricular wall abnormality as shown on echocardiograms. This same group had extensive areas of ischemia discovered during thallium exercise testing, and severe ischemia during 24 to 48 hours of Holter monitoring. These results confirm earlier statistical correlation between measures of anger and hostility and sudden cardiac events.

In a related study, Johns Hopkins University researchers followed up three cohorts of medical students for more than 40 years to determine the association, if any, between anxiety and tension and overall disease-related mortality and premature mortality. Students characterized as tense and anxious with somatic or psychophysiologic manifestations had the highest mortality rate $(20 \%)$, compared with those students classified as stable $(15 \%)$ and volatile but expressive (10\%). Furthermore, not only was the overall mortality of the somatic group higher, but also the deaths occurred at an earlier age than in the other groups.

Whether behavior is an independent risk factor in cardiac disease and overall mortality is another question. Several animal studies have shown that the degree of adverse stimulus necessary to effect cardiac changes is inversely associated with the degree of underlying CAD. Among humans, the degree of the pathologic condition is probably inversely related to the intensity of stimuli necessary to precipitate sudden coronary-related death. Therefore, preven-

(continued on page 63) 